\title{
Capacity-Based Parameter Optimization of Bit-Interleaved Coded CPM With Differential Detection
}

\author{
Rohit Iyer Seshadri, Student Member, IEEE, and Matthew C. Valenti, Senior Member, IEEE
}

\begin{abstract}
The design of bit-interleaved coded continuous phase modulation (CPM) is characterized by the code rate, alphabet size, modulation index, and pulse shape. This paper outlines a methodology for determining the optimal values of these parameters under bandwidth and receiver complexity constraints. The cost function that is used to drive the optimization is the informationtheoretic minimum $\mathcal{E}_{b} / N_{0}$, which is found by evaluating the constrained channel capacity. The capacity can be estimated using Monte Carlo integration. A search for optimal parameters is conducted over a range of coded CPM parameters, bandwidth efficiencies, and channels. To limit complexity and allow any modulation index to be considered, the receiver is constrained to use a soft-output differential phase detector. Bit error rate curves using a binary turbo code confirm that the constrained capacity is a very good indicator of the performance of the complete system.
\end{abstract}

Index Terms-Bit-interleaved coded modulation (BICM), capacity, continuous phase modulation (CPM), differential phase detector.

\section{INTRODUCTION}

$\mathbf{I}$ N RADIO environments suffering from spectral congestion, continuous phase modulation (CPM) [1] is an attractive modulation choice. This is because the small spectral sidelobes of CPM signals can avoid adjacent channel interference. CPM signals also have a constant envelope, which makes them suitable for systems with nonlinear amplifiers. The energy efficiency of CPM can be improved by combining channel coding with CPM, for instance, by using a convolutional code to increase the memory of the modulation [2], [3]. Additional gains in energy efficiency can be made by using ring convolutional codes [4] or capacity-approaching codes instead. However, channel coding generally comes at the expense of bandwidth.

To incorporate coding while holding bandwidth constant, one alternative is to combine a trellis encoder with a CPM modulator that uses a larger alphabet [5], [6]. Systems that use a symbol interleaver to separate the encoder and the modulator are referred to as trellis coded CPM (TCCPM) [7]. Although the interleaver precludes true maximum-likelihood (ML) joint demodulation and decoding, it can be approximated by using

Manuscript received February 17, 2006; revised December 15, 2006 and February 19,2007. The review of this paper was coordinated by Prof. L. Lampe.

The authors are with the Lane Department of Computer Science and Electrical Engineering, West Virginia University, Morgantown, WV 26506-6109 USA (e-mail: iyerseshadri@ieee.org; valenti@ieee.org).

Digital Object Identifier 10.1109/TVT.2007.901078 turbo-style processing [7], [8]. If a bit interleaver is used instead of a symbol interleaver [9], [10], then the symbol sizes of the encoder and the modulator do not need to match, and it is sufficient to use a binary code. The strategy of combining a binary code, a bit-wise interleaver, and a modulator is called bit-interleaved coded modulation (BICM), and, here, we refer to its extension to coded CPM as BICCPM. Not only is BICCPM more convenient to design and implement than TCCPM, but also, results in [7] indicate that BICCPM can provide higher diversity than TCCPM in fading channels, which is consistent with the results for BICM in general [9].

In coded CPM systems, energy and bandwidth efficiency are determined by the alphabet size $M$, the type and width of the pulse shape, the modulation index $h$, the code rate $R_{c}$, and the choice of the demodulator. Our goal in this paper is to provide some insight into the relationship between the energy/ bandwidth tradeoff and the above variables for BICCPM. This is a challenging problem, particularly since there exists an inherent tradeoff between the code rate and CPM parameters. For instance, as the code rate decreases, to maintain a specified spectral efficiency, either a smaller modulation index, a longer partial-response pulse, or a smaller alphabet size must be used. For any particular scenario, it is not clear if the coding gain due to lowering the code rate will offset the performance loss due to using modulation that is further from being orthogonal or the additional ISI due to using longer pulses.

In this paper, we jointly optimize the code rate and CPM parameters for a given bandwidth efficiency and receiver complexity. The cost function is based on the constrained capacity of the system. More specifically, the cost function is the information-theoretic minimum $\mathcal{E}_{b} / N_{0}$ that is required for reliable signaling under the constraints of a class of modulation, a desired bandwidth efficiency, and a particular receiver design. The cost function is evaluated by measuring the average mutual information between the input and the output of an appropriately defined binary composite channel as a function of $\mathcal{E}_{s} / N_{0}$ and then setting $\mathcal{E}_{b} / N_{0}=\left(\mathcal{E}_{s} / N_{0}\right) /\left(R_{c} \log _{2} M\right)$, where $R_{c}$ is set to equal the measured mutual information (i.e., $R_{c}$ is the rate of the binary code that achieves capacity at a particular value of $\left.\mathcal{E}_{s} / N_{0}\right)$.

There are several benefits of performing a capacity-based optimization. The most obvious is that capacity specifies the performance limits for a coded system and inherently takes into account the tradeoff between the code rate and the energy efficiency, which can be easily extended in our case to 
account for the tradeoff between the code rate and modulation parameters. It is a very practical prediction of system performance since either "off-the-shelf" capacity-approaching binary codes or powerful binary codes that are tailored to the specific modulation parameters [11], [12] can be used to get close to the constrained capacity. Although closed-form solutions for constrained capacity are, at times, nontrivial to compute, Monte Carlo integration can be used to estimate the capacity, and by using a very large number of trials, the estimate can be quite accurate [9], [13]. Our capacity calculations also take into account the design of the demodulator. A similar optimization attempt for coherently detected BICCPM was made in [10] using a union bound on the bit error rate (BER) as the cost function. However, [10] limits itself to low constraint length convolutional codes and relatively simple modulation parameters. A key distinction is that the analysis in [10] depends, in part, on the choice of the channel code. In our case, since the information-theoretic limits predicted for a particular combination of code rate and modulation parameters can be (almost) reached using capacity-approaching codes, our analysis is less restricted by the choice of the channel code.

As with any optimization problem, our optimization is also subject to some constraints. The first is on the type of the detector used for CPM. The optimum detector would be coherent [1], which can perfectly track the signal phase and has perfect channel state information. This, however, increases the system complexity. Hence, in this paper, we emphasize differential phase detection [14] of BICCPM signals. Differential detectors base their decisions on the phase differences that are calculated over multiple symbol intervals. The underlying assumption is that any random phase shifts caused by the channel remain constant during the interval over which the phase difference can be calculated, and thus, they can be factored out of the decision metric. Unlike that of coherent detectors, the complexity of the differential detectors does not increase with decreasing $h$, which is a significant design issue at high bandwidth efficiency. Furthermore, our results suggest that the performance of the differential detectors is quite close to the ML coherent receiver at small values of $h$, making them an attractive choice for bandwidth-limited systems. Alternatively, noncoherent detectors [15], [16] can be employed where the information is estimated in the presence of phase uncertainty.

The next constraint is on the search space itself since the parameters involved $\left(R_{c}, h, M\right.$, type, and width of pulse shapes) can assume a wide range of values, thus effectively yielding an infinitely large search space. We hence perform optimization over a trial search space by limiting the allowable values for the above parameters to sensible alternatives.

The rest of this paper is organized as follows. Section II deals with the system model, including a description of softoutput differential detection of CPM signals using what we call the soft-output soft-decision differential phase detector (SO-SDDPD). This detector differs from traditional hardoutput differential detectors [14], [17], [18] and Fonseka's [19] original soft-decision differential phase detector reference, as our detector produces bit-wise log-likelihood ratios (LLRs), which are the required outputs for the composite binary channel over which the mutual information is measured. Section III

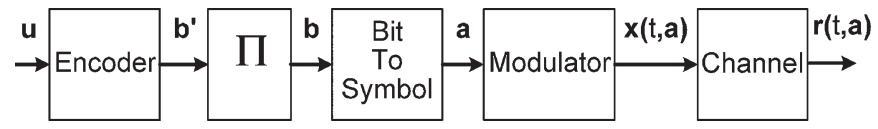

Fig. 1. Block diagram of transmitter and channel.

describes the methodology for finding the capacity under BICCPM constraints. Our optimization process and results are given in Section IV. BER results are given in Section V. Section VI concludes this paper.

\section{System Model}

\section{A. Transmitter}

The transmitter model is shown in Fig. 1. A vector $\mathbf{u}=$ $\left[u_{0}, u_{1}, \ldots, u_{N_{u}-1}\right] \in\{0,1\}^{N_{u}}$ of information bits is passed through a linear binary encoder to produce the codeword $\mathbf{b}^{\prime} \in\{0,1\}^{N_{b}}$. The code rate is $R_{c}=N_{u} / N_{b}$. The row vector $\mathbf{b}^{\prime}$ is then interleaved by multiplying by a permutation matrix $\boldsymbol{\Pi}$ to produce the bit-interleaved codeword $\mathbf{b}=\mathbf{b}^{\prime} \boldsymbol{\Pi}$. The vector $\mathbf{b}$ is arranged in a $\log _{2} M \times N_{a}$ matrix $\mathbf{B}$ with $(i, k)$ element $B_{i, k}=b_{k} \log _{2} M+i$. The number of $M$-ary symbols to be transmitted is $N_{a}=\left\lceil N_{b} / \log _{2} M\right\rceil$. Each column of $\mathbf{B}$ is mapped to one of the $M$ symbols (natural mapping) to produce the vector $\mathbf{a}=\left[a_{0}, a_{1}, \ldots, a_{N_{a}-1}\right] \in$ $\{ \pm 1, \pm 3, \ldots, \pm(M-1)\}^{N_{a}}$, which represents the sequence of coded symbols to be transmitted.

As in [18], the symbol sequence is used to create the following time-varying phase of the transmitted signal:

$$
\varphi(t, \mathbf{a})=\pi h \sum_{i=-\infty}^{\infty} a_{i} q(t-i T)
$$

where $h$ is the modulation index, $T$ is the symbol period, and

$$
q(t)=\int_{-\infty}^{t} g(\tau) d \tau
$$

where $g(t)$ is the pulse shape, which is normalized so that $\int_{-\infty}^{\infty} g(\tau) d \tau=1$. Although the symbol vector a has indexes that range from 0 to $N_{a}-1$, the limits in the summation of (1) are infinite to capture the effect of possible preceding and subsequent coded frames. If there is a sufficient guard time between subsequent frames, as in a packet radio system, then the limits of this summation can be narrowed down to $0 \leq i \leq$ $N_{a}-1$.

Although a wide variety of $g(t)$ may be used, we focus on pulse shapes that ensure that the phase $\varphi(t, \mathbf{a})$ has no discontinuities. One example is to let $g(t)$ be the response of a truncated Gaussian filter to a rectangular pulse of duration $T$, resulting in [18]

$$
g(t)=\frac{1}{T}\left[Q\left(-c B_{g} t\right)-Q\left(-c B_{g}(t-T)\right)\right]
$$

where $B_{g} T$ is the normalized $3-\mathrm{dB}$ bandwidth of the filter, $c=7.546$, and $Q(\cdot)$ is the usual Gaussian $Q$-function. The class of CPM that uses the Gaussian pulse shape of (3) is 
known as Gaussian frequency-shift keying (GFSK). GFSK is a very popular form of CPM and is used in standards such as Global System for Mobile Communications (GSM) and Bluetooth [20].

Another example of pulse shape is the raised cosine (RC) pulse shape [21], i.e.,

$$
g(t)=\frac{1}{L T}\left[1-\cos \left(2 \pi \frac{(t+((L-1) / 2) T)}{L T}\right)\right]
$$

which is nonzero only in the interval $-(((L-1) T) / 2) \leq t \leq$ $(((L+1) T) / 2)$, and $L$ is the width of the pulse shape [the number of symbol intervals for which $g(t)$ is nonzero]. The class of CPM that uses the RC pulse shape of (4) is called $R C-C P M$.

After forming the time-varying phase $\varphi(t, \mathbf{a})$, the transmitter generates the bandpass signal

$$
x(t, \mathbf{a})=\sqrt{2 P_{x}} \cos \left(2 \pi f_{c} t+\varphi(t, \mathbf{a})\right)
$$

which is then transmitted over the radio frequency (RF) channel with power $P_{x}$ and center frequency $f_{c}$. Because the proposed optimization technique operates under a bandwidth constraint, it is important to determine the bandwidth of the transmitted signal. The bandwidth for a fixed data rate is a function of the alphabet size $M$, the modulation index $h$, and the pulse shape $g(t)$, as discussed in [21], and scales by the inverse of the code rate $R_{c}$.

\section{B. Channel}

The modulated signal $x(t, \mathbf{a})$ is transmitted through a frequency nonselective Rician fading channel. The effects of the channel are most conveniently described in terms of the complex envelope of the signal. The complex envelope of the transmitted signal is

$$
\tilde{x}(t, \mathbf{a})=\sqrt{2 P_{x}} \exp (j \varphi(t, \mathbf{a})) .
$$

This signal is multiplied by a complex flat-fading process $\tilde{c}(t)$ and added to white Gaussian noise $\tilde{n}_{w}(t)$, producing the complex envelope of the signal at the input to the receiver, i.e.,

$$
\tilde{r}(t, \mathbf{a})=\tilde{c}(t) \tilde{x}(t, \mathbf{a})+\tilde{n}_{w}(t)
$$

where $\tilde{n}_{w}(t)$ is a complex white Gaussian process with power spectral density $N_{0}$, and

$$
\tilde{c}(t)=\sqrt{P_{s}}+\sqrt{P_{d}} \xi(t)
$$

where $P_{d}$ is the power gain of the diffused component, $P_{s}$ is the power gain of the specular component, and $\xi(t)$ is a circularly symmetric unit power complex Gaussian process. $P_{s}$ and $P_{d}$ are normalized such that $P_{s}+P_{d}=1$. The Rician $K$-factor is given by $K=P_{s} / P_{d}$. When $K=0$, the channel is said to be Rayleigh, and when $K=\infty$, the channel is said to be an additive white Gaussian noise (AWGN). The process $\xi(t)$ is

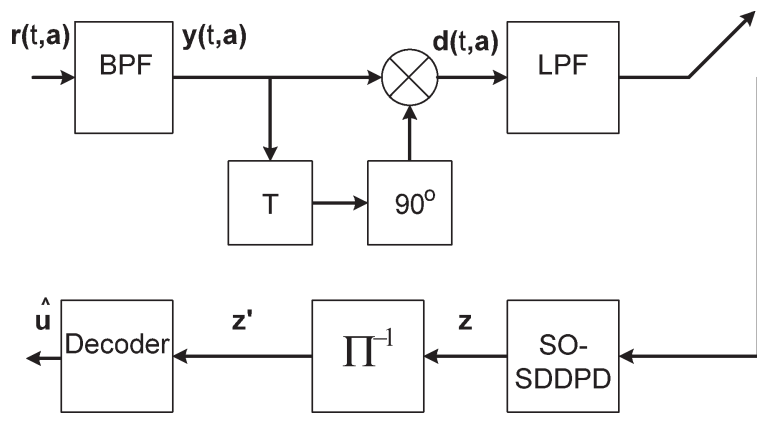

Fig. 2. Receiver block diagram.

usually characterized by an autocorrelation function, which, as an example, could be [22]

$$
R_{\xi}(\tau)=J_{0}\left(2 \pi f_{d} \tau\right)
$$

where $J_{0}$ is the zero-order Bessel function of the first kind, and $f_{d}$ is the maximum Doppler frequency shift.

\section{Differential Front End}

As shown in Fig. 2, the received signal $r(t, \mathbf{a})$, whose complex envelope is given by (7), is passed through a bandpass receive filter that removes the out-of-band noise. It is assumed that the equivalent noise bandwidth of the filter, i.e., $B_{n}$, is greater than the signal's $99 \%$ power bandwidth, and that the passband of the filter is relatively flat. Thus, the informationbearing portion of the signal is essentially undistorted by the filter. Under this assumption, the signal $y(t, \mathbf{a})$ at the output of the bandpass filter has the complex envelope

$$
\begin{aligned}
\tilde{y}(t, \mathbf{a}) & =\tilde{c}(t) \tilde{x}(t, \mathbf{a})+\tilde{n}_{c}(t) \\
& =\sqrt{2 P_{y}(t)} \exp (j \phi(t, \mathbf{a}))
\end{aligned}
$$

where $\tilde{n}_{c}(t)$ is the complex low-pass noise with power $P_{n}=$ $N_{0} B_{n}, P_{y}(t)$ is the power of the received signal, and $\phi(t, \mathbf{a})$ is the phase of the received signal. Due to fading and noise, $P_{y}(t)$ is time varying, although the transmitted signal power $P_{x}$ is constant.

The received phase may be expressed as

$$
\phi(t, \mathbf{a})=\varphi(t, \mathbf{a})+\psi(t)
$$

where $\psi(t)$ is the phase noise as derived in [18], and $\varphi(t, \mathbf{a})$ is as given in (1).

As is shown in Fig. 2, the output of the bandpass filter $y(t, \mathbf{a})$, whose complex envelope is given by (10), is multiplied by a delayed and phase-shifted version of itself. The resulting bandpass signal is

$$
\begin{array}{r}
d(t, \mathbf{a})=-2 \sqrt{P_{y}(t) P_{y}(t-T)} \cos \left(2 \pi f_{c} t+\phi(t, \mathbf{a})\right) \\
\times \sin \left(2 \pi f_{c}(t-T)+\phi(t-T, \mathbf{a})\right) .
\end{array}
$$

Using the well-known trigonometric identity $\cos \alpha \sin \beta=$ $(1 / 2) \sin (\alpha+\beta)-(1 / 2) \sin (\alpha-\beta)$ and dropping the double 
frequency term, after low-pass filtering, $d(t, \mathbf{a})$ becomes

$$
\begin{aligned}
& d_{b}(t, \mathbf{a})=\sqrt{P_{y}(t) P_{y}(t-T)} \\
& \quad \times \sin \left(\phi(t, \mathbf{a})-\phi(t-T, \mathbf{a})+2 \pi f_{c} T\right) .
\end{aligned}
$$

The signal is then sampled at time $t=(k+1) T$, resulting in

$$
\begin{aligned}
d_{b}((k+1) T, \mathbf{a}) & =\sqrt{P_{y}(k T+T) P_{y}(k T)} \\
\times & \sin \left(\phi(k T+T, \mathbf{a})-\phi(k T, \mathbf{a})+2 \pi f_{c} T\right) .
\end{aligned}
$$

As is common in the literature [17], we assume that the design parameter $f_{c} T$ is selected to be an integer, in which case, the received phase differences can be found by computing

$$
\begin{aligned}
\Delta \phi_{k} & =\angle d_{b}((k+1) T, \mathbf{a}) \\
& =\phi(k T+T, \mathbf{a})-\phi(k T, \mathbf{a})
\end{aligned}
$$

where $\angle \kappa \sin z=z$ for any arbitrary constant $\kappa$.

\section{Soft-Output Back End}

In traditional differential detectors such as those described in [14], [17], and [18], hard symbol decisions are made by comparing $\Delta \phi_{k}$, as given in (15), to some threshold. Such a symbol-by-symbol detector is suboptimal for two reasons. First, such symbol-based detectors are unable to adequately compensate for the ISI induced by the partial response CPM signaling. Second, the formation of hard decisions is suboptimal for a channel-coded system since properly implemented soft-decision decoding outperforms hard-decision decoding.

The first drawback can be overcome by modeling the memory in the modulation as a finite-state machine and using the Viterbi algorithm to perform ML demodulation. ML demodulation using the soft phase differences (15) and the Viterbi algorithm was proposed in [19], where it is termed soft-decision differential phase detector with Viterbi decoding (SDDPD-VD). However, the bit decisions made with SDDPD-VD are hard and, therefore, not suitable for softdecision decoding of the channel code.

The second drawback can be alleviated by replacing the Viterbi algorithm in the SDDPD-VD algorithm with the Bahl, Cocke, Jelinek, and Raviv [BCJR or maximum a posteriori] algorithm of [23]. In such cases, the demodulator will output the LLRs of the code bits, i.e.,

$$
\mathcal{Z}_{i, k}=\log \frac{P\left[B_{i, k}=1 \mid \Delta \phi\right]}{P\left[B_{i, k}=0 \mid \Delta \phi\right]}
$$

where $\Delta \phi=\left\{\Delta \phi_{k}, 0 \leq k \leq N_{a}-1\right\}$ is the received sequence of phase differences. In this paper, we use the term softoutput soft-decision differential phase detector (SO-SDDPD) to describe our formulation of the soft differential detector based on the BCJR algorithm, which is the subject of the present section and was previously proposed in [24].

Both the Viterbi and BCJR algorithms require that the underlying modulation be described in terms of a finite-state
TABLE I

$\theta_{i}$ (IN DEGREES) AT DIFFERENT $B_{g} T$ FOR GFSK WITH $h=0.5$

\begin{tabular}{|c|c|c|c|c|c|}
\hline$B_{g} T$ & $\theta_{-2}$ & $\theta_{-1}$ & $\theta_{0}$ & $\theta_{1}$ & $\theta_{2}$ \\
\hline 0.2 & 1.6687 & 20.3893 & 45.8415 & 20.3893 & 1.6687 \\
\hline 0.25 & 0.5443 & 17.9411 & 53.0273 & 17.9411 & 0.5443 \\
\hline 0.5 & 0 & 9.5153 & 70.9684 & 9.5153 & 0 \\
\hline$\infty$ & 0 & 0 & 90 & 0 & 0 \\
\hline
\end{tabular}

TABLE II

$\theta_{i}$ (IN DEgReEs) AT DifFERENT $L$ FOR RC PUlse Shape With $h=0.5$

\begin{tabular}{|c|c|c|c|c|c|}
\hline$L$ & $\theta_{-2}$ & $\theta_{-1}$ & $\theta_{0}$ & $\theta_{1}$ & $\theta_{2}$ \\
\hline 5 & 4.3771 & 23.2035 & 58.6027 & 23.2035 & 4.3771 \\
\hline 3 & 0 & 17.5951 & 54.8098 & 17.5951 & 0 \\
\hline 1 & 0 & 0 & 90 & 0 & 0 \\
\hline
\end{tabular}

machine. For differential detection, the finite states manifest themselves as a finite set of transmitted phase differences, i.e.,

$$
\begin{aligned}
\Delta \varphi_{k}(\mathbf{a}) & =\varphi(k T+T, \mathbf{a})-\varphi(k T, \mathbf{a}) \\
& =\pi h \sum_{i=-\infty}^{\infty} a_{k-i} p_{i}
\end{aligned}
$$

where $\varphi(t, \mathbf{a})$ is as given in (1), and

$$
p_{i}=\int_{i T}^{i T+T} g(t) d t
$$

When the pulse $g(t)$ is not time limited, the number of distinct phase differences will be generally (countably) infinite. However, for pulses of interest, the CPM-induced ISI terms are generally limited to only extend from $Z_{p}$ past symbols to $Z_{f}$ future symbols. In this case, (17) can be written as

$$
\Delta \varphi_{k}(\mathbf{a})=\pi h \sum_{i=-Z_{f}}^{Z_{p}} a_{k-i} p_{i}
$$

and $\Delta \varphi_{k}(\mathbf{a})$ will assume one of only $M^{Z_{p}+Z_{f}+1}$ values. If the Gaussian pulse shape of (3) or the RC pulse shape of (4) is used, then the ISI will be centered such that $Z_{f}=Z_{p}=Z$. Tables I and II list the values of $\theta_{i}=\pi h p_{i}$ for GFSK and RC pulse shapes, respectively, with $h=0.5$. As an example, for GFSK with $B_{g} T=0.5, Z=Z_{f}=Z_{p}=1$.

Soft-output demodulation is performed by running the BCJR algorithm on an $M^{2 Z}$ state trellis. The state at time instant $t=k T$ is given by $S_{k}=\left(a_{k-2 Z}, a_{k-2 Z+1}, \ldots, a_{k-1}\right)$, with $M$ branches emerging out of each state. Once the trellis is so defined, the LLR given by (16) can be decomposed using the BCJR algorithm as

$$
\mathcal{Z}_{i, k}=\log \frac{\sum_{\mathcal{S}^{(1)}} \alpha_{k}\left(s^{\prime}\right) \gamma_{k+1}\left(s^{\prime}, s\right) \beta_{k+1}(s)}{\sum_{\mathcal{S}^{(0)}} \alpha_{k}\left(s^{\prime}\right) \gamma_{k+1}\left(s^{\prime}, s\right) \beta_{k+1}(s)}
$$

where $\mathcal{S}^{(1)}$ is the set of state transitions $\left\{S_{k}=s^{\prime}\right\} \rightarrow\left\{S_{k+1}=s\right\}$ corresponding to $B_{i, k}=+1, \mathcal{S}^{(0)}$ is defined similarly for $B_{i, k}=0$, and $\alpha, \beta$, and $\gamma$ are the metrics in the BCJR algorithm. Because [23] already fully describes how to recursively 
calculate $\alpha$ and $\beta$ from the branch metric $\gamma$, all that remains to completely describe the demodulator is a derivation of $\gamma$.

As in [23], the metric $\gamma_{k+1}\left(s^{\prime}, s\right)$ is defined as

$$
\begin{aligned}
\gamma_{k+1}\left(s^{\prime}, s\right)= & P\left[S_{k+1}=s, \Delta \phi_{k} \mid S_{k}=s^{\prime}\right] \\
= & P\left[S_{k+1}=s \mid S_{k}=s^{\prime}\right] \\
& \times P\left[\Delta \phi_{k} \mid\left(S_{k} \rightarrow S_{k+1}\right)=\left(s^{\prime} \rightarrow s\right)\right]
\end{aligned}
$$

where $\Delta \phi_{k}$ is the received phase difference given in (15). Because the interleaved code bits are equally likely, so are the steady-state branch transition probabilities, and thus, $P\left[S_{k+1}=s \mid S_{k}=s^{\prime}\right]$ is a constant that can be dropped from (21) without affecting the overall LLR (20). Note that if a priori information were delivered from the decoder back to the demodulator, then the a priori information could be used to update the value of $P\left[S_{k+1}=s \mid S_{k}=s^{\prime}\right]$. Such an operation is contemplated in [24] for iterative demodulation and decoding and is outside the scope of this paper.

Let $\Delta \varphi_{k}\left(s^{\prime} \rightarrow s\right)$ be the transmitted phase difference associated with a transition from state $s^{\prime}$ to $s$, calculated by substituting the symbols associated with the two states into (19). Because of this one-to-one correspondence between state transitions and transmitted phase differences, (21) may be rewritten as

$$
\gamma_{k+1}\left(s^{\prime}, s\right)=P\left[\Delta \phi_{k} \mid \Delta \varphi_{k}\left(s^{\prime} \rightarrow s\right)\right]
$$

which is the conditional probability that phase difference $\Delta \phi_{k}$ was received given that phase difference $\Delta \varphi_{k}\left(s^{\prime} \rightarrow s\right)$ was transmitted. Because the received phase difference $\Delta \phi_{k}$ is a continuous random variable, the probability given in (22) is actually zero. It is more appropriate to instead evaluate the probability that $\Delta \phi_{k}$ is within a small region $\left(\varrho^{-}, \varrho^{+}\right)$, i.e.,

$$
\gamma_{k+1}\left(s^{\prime}, s\right)=P\left[\varrho^{-} \leq \Delta \phi_{k}<\varrho^{+} \mid \Delta \varphi_{k}\left(s^{\prime} \rightarrow s\right)\right]
$$

and then let $\epsilon=\varrho^{+}-\varrho^{-} \rightarrow 0$. As $\epsilon$ gets smaller, the above probability becomes

$$
\gamma_{k+1}\left(s^{\prime}, s\right)=\epsilon p_{\Delta \phi}\left(\Delta \phi_{k} \mid \Delta \varphi_{k}\left(s^{\prime} \rightarrow s\right)\right)
$$

where $p_{\Delta \phi}\left(\cdot \mid \Delta \varphi_{k}\left(s^{\prime} \rightarrow s\right)\right)$ is the conditional pdf of the received phase difference given a transmitted phase difference of $\Delta \varphi_{k}\left(s^{\prime} \rightarrow s\right)$. Because $\epsilon$ cancels in the overall LLR (20), it can be dropped, and the branch metric $\gamma_{k+1}\left(s^{\prime}, s\right)$ can be determined by substituting the received phase difference $\Delta \phi_{k}$ into the conditional pdf.

In a practical receiver, one would not want to directly evaluate the true conditional pdf because it is not easily expressed in a closed form. Alternatively, one could precalculate and store the pdf in a lookup table with a finite number of entries and read out the entry closest to the received phase difference. This would be accomplished by partitioning the phase region between 0 and $2 \pi$ into $R$ phase subregions $D_{i}=\left(\varrho_{i}^{-}, \varrho_{i}^{+}\right), 0 \leq$ $i \leq R-1$, where adjacent regions share a common boundary, i.e., $\varrho_{i}^{+}=\varrho_{i+1}^{-}$. The table is read by first determining in which phase region the received phase difference lies and then by outputting the value stored in the table.
Although the above-described technique accurately approximates the true conditional pdf as the size of the phase regions gets smaller, it is suboptimal when a coarser table is used with fewer entries. This is because for wide regions, the conditional pdf within the region is no longer a constant, and therefore, the probability that the received phase difference lies in this region can no longer be approximated by the area of a rectangle, as given by (24). Instead, the probability given by (22) should be calculated by integrating the pdf over the region

$$
\begin{aligned}
P\left[\varrho_{i}^{-} \leq \Delta \phi<\varrho_{i}^{+} \mid \Delta \varphi_{k}\left(s^{\prime} \rightarrow s\right)\right] & \\
& =\int_{\varrho_{i}^{-}}^{\varrho_{i}^{+}} p_{\Delta \phi}\left(\lambda \mid \Delta \varphi_{k}\left(s^{\prime} \rightarrow s\right)\right) d \lambda .
\end{aligned}
$$

The solution to the above integral is given in [18] to be

$$
\begin{aligned}
& P\left[\varrho_{i}^{-} \leq \Delta \phi<\varrho_{i}^{+} \mid \Delta \varphi_{k}\left(s^{\prime} \rightarrow s\right)\right] \\
& \quad=1+F\left(\varrho_{i}^{+} \mid \Delta \varphi_{k}\left(s^{\prime} \rightarrow s\right)\right)-F\left(\varrho_{i}^{-} \mid \Delta \varphi_{k}\left(s^{\prime} \rightarrow s\right)\right)
\end{aligned}
$$

when $\varrho_{i}^{-} \leq \Delta \varphi_{k}\left(s^{\prime} \rightarrow s\right)<\varrho_{i}^{+}$, and

$$
\begin{aligned}
P\left[\varrho_{i}^{-}\right. & \left.\leq \Delta \phi<\varrho_{i}^{+} \mid \Delta \varphi_{k}\left(s^{\prime} \rightarrow s\right)\right] \\
& =F\left(\varrho_{i}^{+} \mid \Delta \varphi_{k}\left(s^{\prime} \rightarrow s\right)\right)-F\left(\varrho_{i}^{-} \mid \Delta \varphi_{k}\left(s^{\prime} \rightarrow s\right)\right)
\end{aligned}
$$

otherwise. The function $F$ is described by the set of equations given in [18]. Because the function $F$ depends on the Rician $K$-factor and the ratio $\mathcal{E}_{s} / N_{0}$, it is assumed that these quantities are known to the receiver or that that receiver can estimate them. The SDDPD does not, however, require estimates of the fading amplitudes.

The SDDPD works by first precalculating $P\left[\varrho_{i}^{-} \leq \Delta \phi<\right.$ $\left.\varrho_{i}^{+} \mid \Delta \varphi_{k}\left(s^{\prime} \rightarrow s\right)\right]$ for each state transition $\left(s^{\prime} \rightarrow s\right)$ and each phase region $D_{i}$ using (26) and (27). The results are then stored in a table with $R$ columns (one for each phase region) and $M^{2 Z+1}$ rows (one for each state transition). The table only needs to be generated once for a particular SNR and Rician $K$-factor, but needs to be recreated as these quantities change. For each received phase difference $\Delta \phi_{k}$, the receiver determines the index of the phase region $D_{i}$ in which it lies, i.e., the value of $i$ for which $\varrho_{i}^{-} \leq \Delta \phi_{k}<\varrho_{i}^{+}$. This index is then used to read out the $i$ th column from the table, whose elements are used as the branch metrics $\gamma_{k+1}\left(s^{\prime}, s\right)$ for the corresponding state transitions $\left(s^{\prime} \rightarrow s\right)$. Once the metrics are determined for every branch in the trellis, the demodulator executes the BCJR algorithm using (20), producing the LLR $\mathcal{Z}_{i, k}$ for each bit $i$ of each symbol $k$. The LLRs are then placed into a row vector $\mathbf{z}$ such that $z_{k} \log _{2} M+i=\mathcal{Z}_{i, k}$. The vector is then deinterleaved, and the resulting sequence $\mathbf{z}^{\prime}$ is fed to the channel decoder for soft-decision decoding.

\section{CAPACITY UNDER BICCPM AND DEMODULATOR CONSTRAINTS}

The BICCPM system shown in Figs. 1 and 2 can be viewed as shown in Fig. 3. The composite channel consists of the modulator, the channel, and the soft-output demodulator. The 


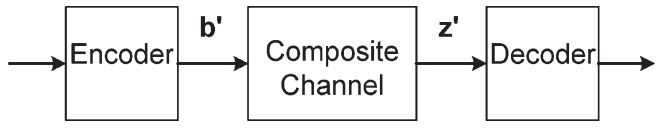

Fig. 3. BICM system represented using a composite channel model. The composite channel includes the modulator, the channel, and the demodulator.

constrained capacity is found by evaluating the average mutual information between the input and the output of this composite channel. As mutual information is a measurable quantity, it can be estimated using Monte Carlo techniques in conjunction with a simulation of the actual demodulator, much the same way that extrinsic information transfer charts are generated [25].

As discussed in [9], BICM transforms the composite channel into $\log _{2} M$ parallel binary-input continuous-output channels, which we call BICM subchannels. With a sufficiently large random bit interleaver between the encoder and the modulator, these subchannels will be independent. Because the capacity of parallel channels adds, the capacity of the overall BICM system is

$$
C=\sum_{i=1}^{\log _{2} M} C_{i}
$$

where $C_{i}$ is the capacity of the $i$ th BICM subchannel. The capacity $C_{i}$ is the average mutual information that is measured between input $b_{i}^{\prime} \in\{0,1\}$ and the corresponding LLR $z_{i}^{\prime}$ at the output of the $i$ th BICM subchannel, i.e.,

$$
C_{i}=I\left(b_{i}^{\prime} ; z_{i}^{\prime}\right)=E\left[i\left(b_{i}^{\prime} ; z_{i}^{\prime}\right)\right] .
$$

Since $b^{\prime}$ is discrete, the mutual information random variable $i(b ; z)$ can be written as [26]

$$
i(b ; z)=\log \frac{1}{P[b]}+\log P[b \mid z] .
$$

Assuming that $b$ is equally likely to be 1 or $0,1 / P[b]=2$. From the mathematics of LLRs, we may write $\log P[b \mid z]=$ $-\max *\left(0, z(-1)^{b}\right)$, where $\max *$ is as defined in [27]. Substituting (28) and (29) with $i(b ; z)$, as defined in (30), gives the expression for BICCPM capacity, i.e.,

$$
C=\sum_{i=1}^{\log _{2} M}\left(\log 2-E\left[\max *\left(0, z_{i}^{\prime}(-1)^{b_{i}^{\prime}}\right)\right]\right)
$$

which is in units of nats. To convert to bits, (31) must be divided by $\log 2$.

Since the capacity is found by measuring the mutual information between the modulator input and the soft demodulator output, it takes into account the constraints of not only the modulation and the channel but also the constraints imposed by the receiver formulation. This allows receiver design issues such as the choice of phase regions to be studied in terms of the impact on constrained capacity. In fact, other receiver designs such as the coherent receiver of [10] can be characterized in terms of constrained capacity. All that is required is for the demodulator to produce an LLR.

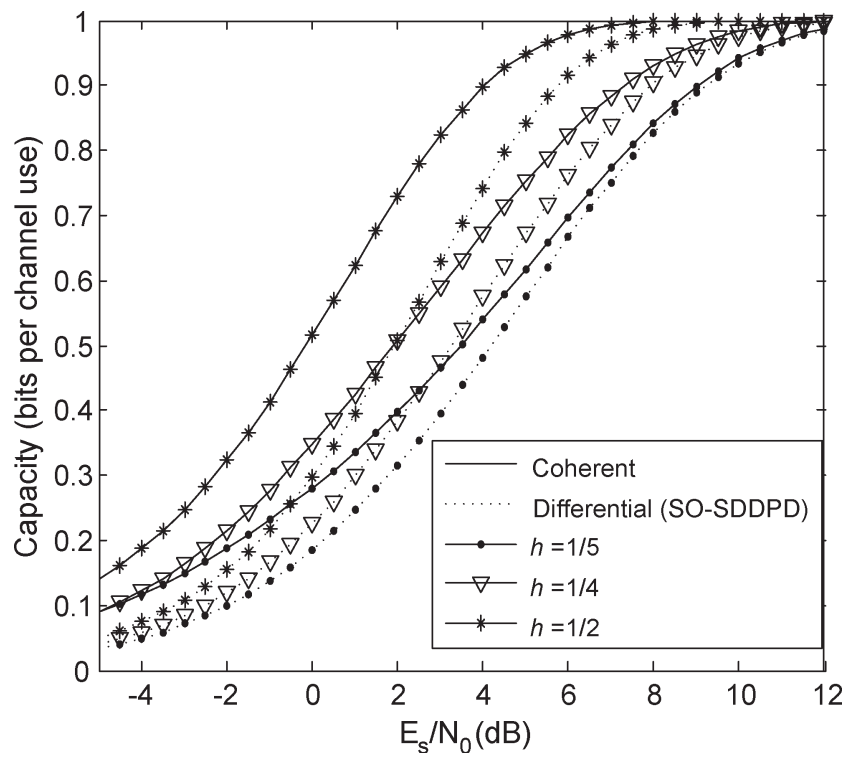

Fig. 4. BICCPM capacity versus $\mathcal{E}_{s} / N_{0}(\mathrm{~dB})$ in AWGN. The modulation parameters are $M=2, B_{g} T=\infty$, and $h \in\{1 / 2,1 / 4,1 / 5\}$. (Dotted curves) Capacity using SO-SDDPD. (Solid curves) Capacity using a coherent detector.

Due to the high dimensionality of the problem and the nonlinear equations that are involved, neither a closed-form expression nor numerical integration is a feasible method to find (31). Fortunately, (31) can be evaluated through Monte Carlo integration using a large number of trials. As in [13], we found that simulating about 1 million symbols per SNR point was sufficient to estimate the true capacity with a high degree of confidence.

Example capacity curves are shown for an AWGN channel in Fig. 4. Fig. 4 shows the BICCPM-constrained capacity $C$ versus $\mathcal{E}_{s} / N_{0}$ for binary $(M=2)$ full response (GFSK with $\left.B_{g} T=\infty\right)$ signaling with $h \in\{1 / 5,1 / 4,1 / 2\}$. The figure shows the constrained capacity of not only the SO-SDDPD demodulator discussed in Section II-D but also the capacity using the soft-output coherent demodulator of [10].

It is interesting to note that the largest gap between the capacities of the two demodulators is about $3 \mathrm{~dB}$, which occurs for the full-response case (Fig. 4) with the highest value of $h(h=1 / 2)$. However, the gap closes with diminishing $h$. It should also be noted that whereas the trellis complexity of the differential detector does not depend on the choice of $h$, the complexity of the coherent detector increases with increasing $n$, where $h=k / n$. In fact, if $h$ cannot be expressed as a rational fraction, then a finite complexity coherent detector cannot be formulated. Since we are interested in bandwidth-efficient systems with low values of $h$, the combination of reduced complexity and only moderate performance loss compared to coherent detection makes the proposed differential detection scheme very attractive.

\section{CAPacity-Based Selection of CPM Parameters}

Given any choice of modulation parameters $[h, M$, and $g(t)]$, a receiver design, and a channel, the capacity $C$ (in units of nats) is found using (31). For convenience, $C$ is normalized as $\bar{C}=$ $C / \log M$. The SNR evaluated at a particular code rate $R_{c}=\bar{C}$ 
is the minimum $\mathcal{E}_{s} / N_{0}$ required for reliable signaling at that $R_{c}$. The corresponding minimum required $\mathcal{E}_{b} / N_{0}$ can then be found using $\mathcal{E}_{s}=R_{c} \log _{2} M \mathcal{E}_{b}$. As the parameters change, so does the minimum value of $\mathcal{E}_{b} / N_{0}$, and what we seek is to determine its global minimum for all possible values of coded modulation parameters for the particular channel and receiver.

We could allow the set $\left(h, M, g(t), R_{c}\right)$ to assume any value. However, the resulting solution could have a high bandwidth (i.e., a low $R_{c}$ or a high $h$ ), or it could result in a high complexity receiver [i.e., a $g(t)$ with a large value of $Z$ ]. Hence, we restrict the search space to reasonable solutions and impose bandwidth as well as complexity constraints. The complexity constraint requires that the receiver be differential (SO-SDDPD) and contain no more than $M^{2}$ states and restrict $M \leq 4$. Because it is differential, the receiver's complexity will not depend on the choice on $h$, and since it contains $M^{2}$ states, only adjacent symbol interference is mitigated $(Z=1)$. Although pulse shapes with ISI that extends beyond the adjacent symbols $(Z>1)$ are allowed in the search space, any ISI beyond $Z=1$ will degrade performance.

With a bandwidth constraint, $h, M, g(t)$, and $R_{c}$ become related. As in [10], the bandwidth constraint is expressed in terms of the coded normalized double-sided $99 \%$ power bandwidth $2 B_{99} T_{b}$, where $T_{b}$ is the information bit period. To find $2 B_{99} T_{b}$, first, the bandwidth of the uncoded signal is found as a function of $h, M$, and the pulse shaping parameter (e.g., $B_{g} T$ or $L$ ) using methods given in [21]. The uncoded bandwidth is simply multiplied by $1 / R_{c}$ to determine the coded bandwidth. Thus, with a given $2 B_{99} T_{b}$, we are free to pick three of the four parameters ( $h, M, B_{g} T / L$, and $R_{c}$ ); however, the fourth parameter will be limited by some minimum (or maximum) value.

The pulse shapes may be either GFSK with parameters $B_{g} T=0.5,0.25$, and 0.2 or RC with $L=3$ or 5 . We consider the code rates $R_{c}=\{6 / 7,5 / 6,3 / 4,2 / 3,1 / 2,1 / 3,1 / 4,1 / 5\}$. We limit the alphabet size $M$ to be either 2 or 4 . The receiver is restricted to use SO-SDDPD with uniformly spaced phase regions with $R=40$ for $M=2$ and $R=26$ for $M=4$. Finally, we consider $2 B_{99} T_{b}=\{0.4,0.6,0.8,0.9,1.0,1.2\}$. The value of $h$ is determined by the choice of the other parameters along with the bandwidth constraint. The optimization is run for a Rayleigh channel $(K=0)$ and a Rician channel with $K=6 \mathrm{~dB}$. There are 80 design points to consider for each of the six values of $2 B_{99} T_{b}$ and two channels. For each design point, $2 B_{99} T_{b}$, and channel, $C$ and, consequently, the theoretic minimum $\mathcal{E}_{b} / N_{0}$ were found. Then, for a particular channel and $2 B_{99} T_{b}$, the design point with the smallest minimum $\mathcal{E}_{b} / N_{0}$ was selected and declared the best design for that channel and bandwidth.

Because a total of 960 capacity curves were generated, a full account of the results cannot be given in this paper. Figs. 5 and 6 show representative intermediate results. In Fig. 5, results are shown in Rician fading $(K=6 \mathrm{~dB})$ for the subset of design points that contain $R_{c}=2 / 3$ and GFSK pulse shapes. For each of the six design points, a curve is given, showing the theoretic required $\mathcal{E}_{b} / N_{0}$ as a function of $2 B_{99} T_{b}$. Also listed is the value of $h$ for the curve with the smallest required $\mathcal{E}_{b} / N_{0}$. For all but the largest $2 B_{99} T_{b}$, the design point $M=4$ and

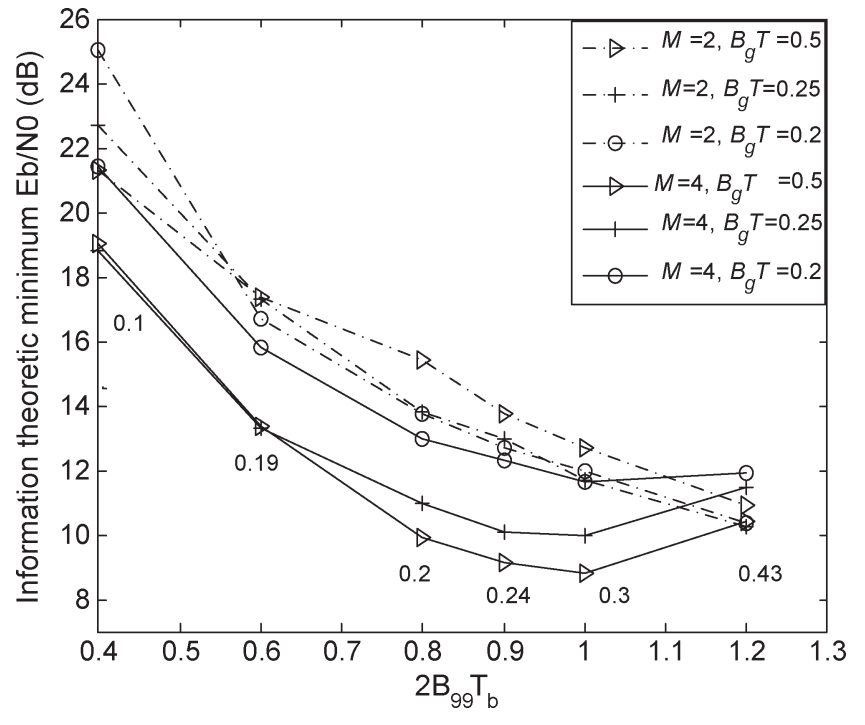

Fig. 5. Minimum required $\mathcal{E}_{b} / N_{0}$ as a function of normalized bandwidth $2 B_{99} T_{b}$ for BICCPM in Rician fading $(K=6 \mathrm{~dB})$. The code rate is $R_{c}=2 / 3$, GFSK modulation used with $M=\{2,4\}$, and demodulation is implemented using SO-SDDPD. The numbers denote modulation indexes corresponding to GFSK parameters with the lowest $\min \left\{\mathcal{E}_{b} / N_{0}\right\}$ at different $2 B_{99} T_{b}$

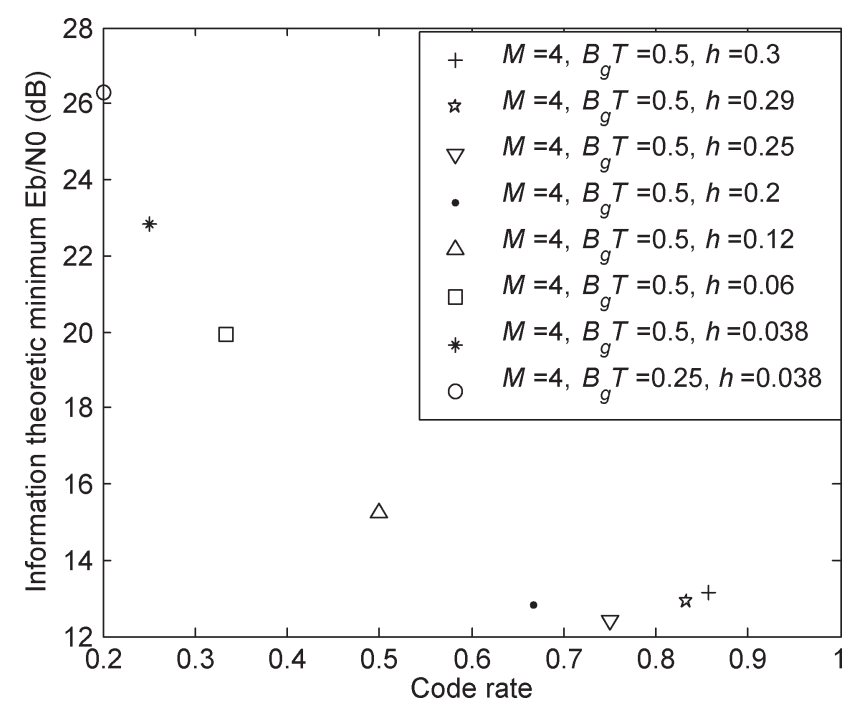

Fig. 6. Minimum required $\mathcal{E}_{b} / N_{0}$ as a function of the code rate for BICCPM with GFSK signaling in Rayleigh fading with SO-SDDPD and under bandwidth constraint $2 B_{99} T_{b}=0.8$. The legend specifies the GFSK parameters $(M, h$, $\left.B_{g} T\right)$ that achieves this minimum. Under the given constraints, the design $\left\{R_{c}=3 / 4, M=4, h=0.25, B_{g} T=0.5\right\}$ has the best energy efficiency.

$B_{g} T=0.5$ has the lowest $\mathcal{E}_{b} / N_{0}$. In Fig. 6, the bandwidth is held fixed at $2 B_{99} T_{b}=0.8$ in a Rayleigh fading channel, and the minimum $\mathcal{E}_{b} / N_{0}$ for each code rate is shown. From the curve, it is apparent that $R_{c}=3 / 4$ is the best code rate for this $2 B_{99} T_{b}$, along with the parameters $M=4, h=0.25$, and $B_{g} T=0.5$. Fig. 6 also shows the tradeoff between the code rate and CPM parameters at a fixed bandwidth. As an example, when $R_{c}$ is lowered from $6 / 7$ to $3 / 4$, an improvement in the energy efficiency is seen due to increased coding gain. However, when $R_{c}$ is lowered below $3 / 4$, the scaling of CPM parameters (primarily $h$ ) not only offsets any potential coding gain but, in fact, also worsens the performance. The design parameters 
TABLE III

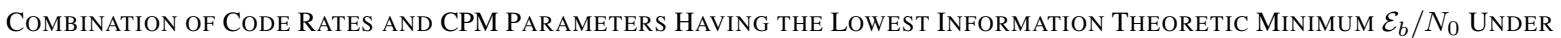
THE CONSTRAint OF USIng SO-SDDPD In RAYLEIGH AND Rician FADING $(K=6 \mathrm{~dB})$ AT DifFeRENT $2 B_{99} T_{b}$

\begin{tabular}{|c|c|c|c|c|c|c|c|}
\hline Channel & $2 B_{99} T_{b}$ & Rate & $M$ & Pulse shape & $\left\{L, B_{g} T\right\}$ & $h$ & $\min \left\{\mathcal{E}_{b} / N_{0}\right\} \mathrm{dB}$ \\
\hline Rayleigh & 0.4 & $5 / 6$ & 4 & GFSK & 0.25 & 0.14 & $20.24 \mathrm{~dB}$ \\
\cline { 2 - 8 } & 0.6 & $5 / 6$ & 4 & GFSK & 0.25 & 0.26 & $14.92 \mathrm{~dB}$ \\
\cline { 2 - 8 } & 0.8 & $3 / 4$ & 4 & GFSK & 0.5 & 0.25 & $12.38 \mathrm{~dB}$ \\
\cline { 2 - 8 } & 0.9 & $2 / 3$ & 4 & GFSK & 0.5 & 0.24 & $11.99 \mathrm{~dB}$ \\
\cline { 2 - 8 } & 1.0 & $2 / 3$ & 4 & GFSK & 0.5 & 0.3 & $11.44 \mathrm{~dB}$ \\
\cline { 2 - 8 } & 1.2 & $6 / 7$ & 2 & RC & 3 & 0.73 & $10.97 \mathrm{~dB}$ \\
\hline Rician & 0.4 & $6 / 7$ & 4 & GFSK & 0.5 & 0.097 & $16.52 \mathrm{~dB}$ \\
\cline { 2 - 8 } & 0.6 & $5 / 6$ & 4 & GFSK & 0.5 & 0.18 & $11.67 \mathrm{~dB}$ \\
\cline { 2 - 8 } & 0.8 & $5 / 6$ & 4 & GFSK & 0.5 & 0.29 & $9.09 \mathrm{~dB}$ \\
\cline { 2 - 8 } & 0.9 & $3 / 4$ & 4 & GFSK & 0.5 & 0.285 & $8.87 \mathrm{~dB}$ \\
\cline { 2 - 8 } & 1.0 & $2 / 3$ & 4 & GFSK & 0.5 & 0.3 & $8.83 \mathrm{~dB}$ \\
\cline { 2 - 7 } & 1.2 & $6 / 7$ & 2 & RC & 3 & 0.73 & $8.32 \mathrm{~dB}$ \\
\hline
\end{tabular}

that minimize $\mathcal{E}_{b} / N_{0}$ for each bandwidth constraint and the corresponding value of the minimum $\mathcal{E}_{b} / N_{0}$ are tabulated in Table III for Rayleigh fading and for Rician fading. As can be seen from the table, GFSK outperforms RC-CPM, except at the most relaxed bandwidth constraint. Further insight into the performance of the SO-SDDPD with different pulse shapes can be obtained from Tables I and II. $B_{g} T=\infty$ and $L=1$ indicate full-response CPM $\left(Z_{f}=Z_{p}=0\right)$. As $B_{g} T$ is lowered or $L$ is increased, the amount of ISI increases. It is interesting to note that for a given pulsewidth, the amount of ISI in the RC pulse shape is greater than GFSK. As a consequence, for the same $M$, $h$, and pulsewidth, RC-CPM would have a more compact power spectrum than GFSK. This also implies that at a fixed $2 B_{99} T_{b}$, $R_{c}, M$, and pulsewidth, RC-CPM allows us to have a higher value of $h$. Typically, larger values of $h$ result in lower values of $\min \left\{\mathcal{E}_{b} / N_{0}\right\}$; however, since the induced ISI is also greater, the RC-CPM may not always have better energy efficiency.

\section{Performance of an Actual Coded System}

BER simulations were performed for the proposed BICCPM system using the Universal Mobile Telecommunications System turbo code [28] and SO-SDDPD detection. The codeword length was $N_{b}=6720$ bits, and the decoder performed 16 decoder iterations. Although the mother code rate is $R_{c}=1 / 3$, rate matching was performed to obtain higher code rates. Enough trials were run at each $\mathcal{E}_{b} / N_{0}$ to $\log 30$ frame errors.

Simulations were performed for both Rayleigh fading and Rician fading with $K=6 \mathrm{~dB}$. In each case, the optimal parameters were chosen from Table III for $2 B_{99} T_{b}=0.9$. The BER in Rayleigh fading for 4-GFSK with parameters $h=0.24$, $B_{g} T=0.5$, and $R_{c}=2 / 3$ is shown in Fig. 7. From this curve, it is seen that the $\mathcal{E}_{b} / N_{0}$ required to achieve a BER of $10^{-5}$ is $12.93 \mathrm{~dB}$, which is less than $1 \mathrm{~dB}$ away from the $\mathcal{E}_{b} / N_{0}$ of $11.99 \mathrm{~dB}$ predicted by Table III. Space constraints prevent us from displaying a similar plot for Rician fading. However, in both cases, BER simulation results confirm that constrained capacity is a practical measure of BICCPM performance since it is possible to signal within $1 \mathrm{~dB}$ of the capacity by using offthe-shelf binary capacity-approaching codes.

It is also informative to compare the performance of the coded system against that of an uncoded system with the same spectral efficiency. Simulation of uncoded CPM using SDDPD detection and binary GFSK with $h=0.5$ and $B_{g} T=0.3$ is

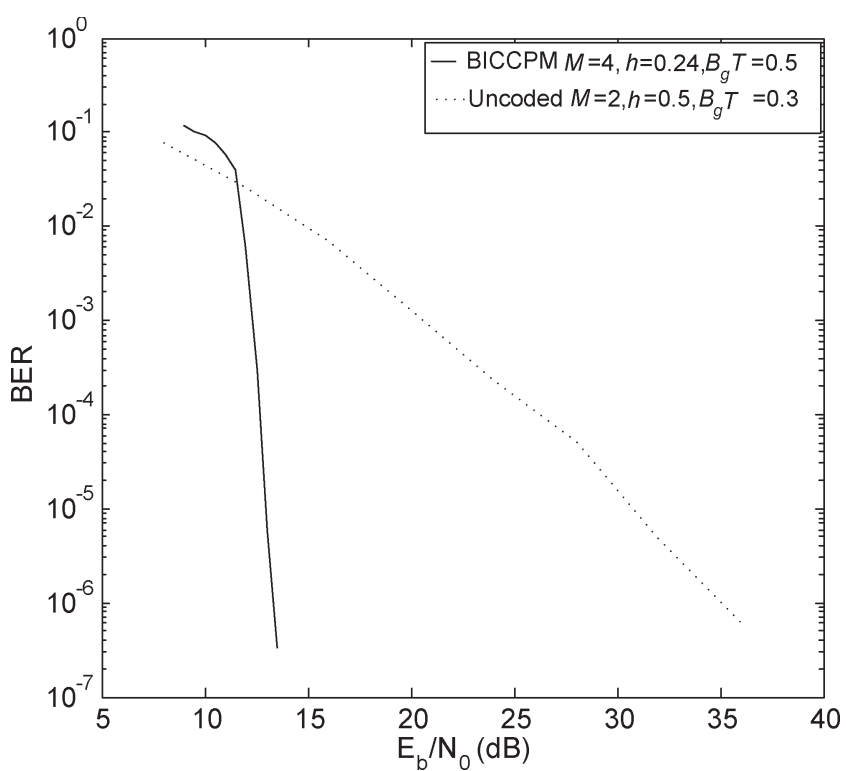

Fig. 7. BER of (solid line) coded and (dotted line) uncoded GFSK in Rayleigh fading under bandwidth constraint $2 B_{99} T_{b}=0.9$ using SO-SDDPD. The coded (BICCPM) system uses a rate $R_{c}=2 / 3$, length $N_{b}=6720$ turbo code, 16 decoder iterations, $R=26$ phase regions, and GFSK parameters $M=4$, $h=0.24$, and $B_{g} T=0.5$. The uncoded system uses $R=40$ phase regions and GFSK parameters $M=2, h=0.5$, and $B_{g} T=0.3$.

shown in Fig. 7 for the Rayleigh channel. These parameters were chosen because they are used in the GSM standard and because they also result in $2 B_{99} T_{b}=0.9$. It can be seen that the coding gain at $\mathrm{BER}=10^{-5}$ is $16 \mathrm{~dB}$.

\section{CONCLUSiON}

We have investigated the problem of designing bitinterleaved coded CPM systems under bandwidth constraints. A methodology for solving the above problem using the constrained capacity as the cost function has been outlined. Monte Carlo integration with a large number of trials offers a computationally feasible method for determining the capacity for a wide range of coded CPM parameters and detector designs. Our BER results demonstrate that the information-theoretic limits predicted by the capacity are practical indicators of the system performance due to the availability of capacity-approaching binary codes. To limit the receiver complexity, a soft-output soft-decision differential phase detector has been developed for 
detecting the CPM signals. Our proposed methodology is used to perform optimization over a trial search space.

Our approach to finding the most energy-efficient combination of code rates and CPM parameters at different bandwidth efficiencies is a "brute force" approach, which is very computationally intensive. As an example, nearly 1000 different capacity curves had to be generated and analyzed to create Table III. A more computationally efficient approach to the optimization would be to apply simulated annealing [29] by treating the constrained capacity as the internal energy and defining the state as the combination of the code rate and CPM parameters. An iterative type of search could also be used by running a few Monte Carlo trials over a coarse search space and then "zooming in" on the parameters of interest and running further trials. Such improved optimization techniques remain topics for further study.

\section{ACKNOWLEDGMENT}

The authors would like to thank Dr. J. Fonseka, Dr. D. Lao, Dr. C. Kwan, the anonymous reviewers, and the Associate Editor for their valuable assistance regarding this paper.

\section{REFERENCES}

[1] J. B. Anderson, T. Aulin, and C. E. Sundberg, Digital Phase Modulation. New York: Plenum, 1986.

[2] S. V. Pizzi and S. G. Wilson, "Convolutional coding combined with continuous phase modulation," IEEE Trans. Commun., vol. COM-33, no. 1, pp. 20-29, Jan. 1985.

[3] T. Svensson and A. Svensson, "On convolutionally encoded partial response CPM," in Proc. IEEE Veh. Technol. Conf., Amsterdam, The Netherlands, Sep. 1999, pp. 663-667.

[4] B. Rimoldi and Q. Li, "Coded continuous phase modulation using ring convolutional codes," IEEE Trans. Commun., vol. 43, no. 11, pp. 27142720, Nov. 1995.

[5] R. W. Kerr and P. J. McLane, "Coherent detection of interleaved trellis encoded CPFSK on shadowed mobile satellite channels," IEEE Trans. Veh. Technol., vol. 41, no. 2, pp. 159-169, May 1992.

[6] L. Yiin and G. L. Stüber, "Noncoherently detected trellis-coded partial response CPM on mobile radio channels," IEEE Trans. Commun., vol. 44, no. 8, pp. 967-975, Aug. 1996.

[7] K. R. Narayanan and G. L. Stüber, "Performance of trellis-coded CPM with iterative demodulation and decoding," IEEE Trans. Commun., vol. 49, no. 4, pp. 676-687, Apr. 2001.

[8] M. Xiao and T. Aulin, "Serially concatenated continuous phase modulation with convolutional codes over rings," IEEE Trans. Commun., vol. 54, no. 8, pp. 1387-1396, Aug. 2006.

[9] G. Caire, G. Taricco, and E. Biglieri, "Bit-interleaved coded modulation," IEEE Trans. Inf. Theory, vol. 44, no. 3, pp. 927-946, May 1998.

[10] P. Moqvist and T. Aulin, "Serially concatenated continuous phase modulation with iterative decoding," IEEE Trans. Commun., vol. 49, no. 11, pp. 1901-1915, Nov. 2001.

[11] K. R. Narayanan, I. Altunbaş, and R. S. Narayanaswami, "Design of serially concatenated MSK schemes based on density evolution," IEEE Trans. Commun., vol. 51, no. 8, pp. 1283-1295, Aug. 2003.

[12] M. Xiao and T. Aulin, "Irregular repeat continuous phase modulation," IEEE Commun. Lett., vol. 9, no. 8, pp. 723-725, Aug. 2005.

[13] S. J. MacMullan and O. M. Collins, "The capacity of orthogonal and bi-orthogonal codes on the Gaussian channel," IEEE Trans. Inf. Theory, vol. 44, no. 3, pp. 1217-1232, May 1998.

[14] M. K. Simon and C. C. Wang, "Differential detection of Gaussian MSK in a mobile radio environment," IEEE Trans. Veh. Technol., vol. VT-33, no. 3, pp. 307-320, Nov. 1984.

[15] G. Colavolpe and R. Raheli, "Noncoherent sequence detection of continuous phase modulations," IEEE Trans. Commun., vol. 47, no. 9, pp. 13031307, Sep. 1999.

[16] L. Lampe, R. Schober, and M. Jain, "Noncoherent sequence detection receiver for Bluetooth systems," IEEE J. Sel. Areas Commun., vol. 23, no. 9, pp. 1718-1727, Sep. 2005.
[17] A. Yongaçoḡlu, D. Makrakis, and K. Feher, "Differential detection of GMSK using decision feedback," IEEE Trans. Commun., vol. 36, no. 6, pp. 641-649, Jun. 1988.

[18] I. Korn, "GMSK with differential phase detection in the satellite mobile channel," IEEE Trans. Commun., vol. 38, no. 11, pp. 1980-1986, Nov. 1990.

[19] J. P. Fonseka, "Soft-decision differential phase detection with Viterbi decoding in satellite mobile systems," J. Commun. Netw., vol. 3, no. 3, pp. 265-272, Sep. 2001.

[20] Bluetooth Special Interest Group, Specification of the Bluetooth system, Nov. 2003. Core Version 1.2.

[21] J. Proakis, Digital Communications, 4th ed. New York: McGraw-Hill, 2001

[22] W. C. Jakes, Microwave Mobile Communications. New York: Wiley, 1974

[23] L. Bahl, J. Cocke, F. Jelinek, and J. Raviv, "Optimal decoding of linear codes for minimizing symbol error rate," IEEE Trans. Inf. Theory, vol. IT-20, no. 2, pp. 284-287, Mar. 1974.

[24] R. I. Seshadri and M. C. Valenti, "Soft-in/soft-out noncoherent sequence detection for Bluetooth: Capacity, error rate and throughput analysis," in Proc. IEEE Wireless Commun. Netw. Conf., Las Vegas, NV, Apr. 2006, pp. 1354-1359.

[25] S. ten Brink, "Convergence behavior of iteratively decoded parallel concatenated codes," IEEE Trans. Commun., vol. 49, no. 10, pp. 1727-1737, Oct. 2001.

[26] R. G. Gallager, Information Theory and Reliable Communications. New York: Wiley, 1968.

[27] A. Viterbi, "An intuitive justification and a simplified implementation of the MAP decoder for convolutional codes," IEEE J. Sel. Areas Commun., vol. 16, no. 2, pp. 260-264, Feb. 1998.

[28] "Universal mobile telecommunications system," Multiplexing and Channel Coding (FDD), Sep. 2005. 3GPP2 TS 125.212 Version 6.6.0.

[29] S. Kirkpatrick, C. D. Gelatt, Jr., and M. P. Vecchi, "Optimization by simulated annealing," Science, vol. 220, no. 4598, pp. 671-680, May 1983.

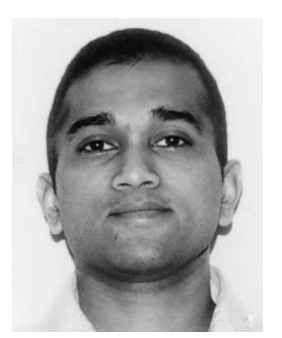

Rohit Iyer Seshadri (S'03) received the M.S. degree in electrical engineering from The Pennsylvania State University, University Park, in 2003. He is currently working toward the $\mathrm{Ph} . \mathrm{D}$. degree in electrical engineering at the Lane Department of Computer Science and Electrical Engineering, West Virginia University, Morgantown.

His current research interests include the areas of wireless communication systems and error correction coding.

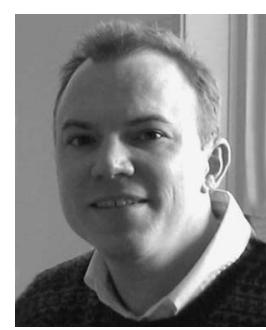

Matthew C. Valenti (M'99-SM'07) received the B.S.E.E. degree from the Virginia Polytechnic Institute and State University (Virginia Tech), Blacksburg, in 1992, the M.S.E.E. degree from The Johns Hopkins University, Baltimore, MD, in 1995, and the Ph.D. degree in electrical engineering from Virginia Tech in 1999, where he was a Bradley Fellow.

Prior to attending the graduate school at Virginia Tech, he was an Electronics Engineer at the United States Naval Research Laboratory, Washington, DC. $\mathrm{He}$ is currently an Associate Professor with the Lane Department of Computer Science and Electrical Engineering, West Virginia University, Morgantown. $\mathrm{He}$ also acts as a consultant to several companies engaged in various aspects of turbo codec design, including software radio, FPGA, and ASIC implementations for military, satellite, and third-generation cellular applications. His research interests include the areas of communication theory, error correction coding, applied information theory, and wireless multiple-access networks.

Dr. Valenti serves as an Associate Editor for the IEEE TRANSACTIONS ON VEHICULAR TECHNOLOGY and has been on the technical program committee for several international conferences. 